\title{
The brightness of SN 1991T and the uniformity of decline-rate and colour corrected absolute magnitudes of supernovae la*
}

\author{
T. Richtler ${ }^{1}$, J. B. Jensen ${ }^{2, \star \star}$, J. Tonry ${ }^{3}$, B. Barris ${ }^{3}$, and G. Drenkhahn ${ }^{4}$ \\ 1 Universidad de Concepción, Departamento de Física, Casilla 160-C, Concepción, Chile \\ 2 Gemini Observatory, 670 N. Aòhoku Place Hilo, HI 96720, USA \\ e-mail: jjensen@gemini.edu \\ 3 Institute for Astronomy, University of Hawaii 2680 Woodlawn Drive, Honolulu, HI 96822, USA \\ 4 Max-Planck-Institut für Astrophysik, Postfach 1317, 85741 Garching bei München, Germany \\ e-mail: georg@mpa-garching.mpg.de
}

Received 7 August 2000 / Accepted 14 November 2000

\begin{abstract}
We present a distance to NGC 4527, the host galaxy of the type Ia SN 1991T, measured by surface brightness fluctuations. This supernova has been labelled "peculiar" both on the grounds of its spectroscopic behaviour and its apparent overluminosity with respect to other supernovae. The distance modulus to NGC 4527 and thus to SN $1991 \mathrm{~T}$ is $30.26 \pm 0.09$. This relatively short distance largely removes the discrepancy with other Ia supernovae having similar light-curve characteristics and also removes the motivation for interpreting SN 1991T as a super-Chandrasekhar explosion. However, the reddening uncertainty results in significant uncertainty of the absolute magnitudes. We show further that, although the intrinsic absolute magnitudes among Ia supernovae are quite different, their brightness, corrected for decline-rate and colour, shows a high degree of uniformity, including underluminous events like SN 1991bg and SN 1997cn. In particular, the present data do not support a significant difference between corrected SNe Ia luminosities in late-type and early-type host galaxies.
\end{abstract}

Key words. supernovae: general - supernovae: individual: SN 1991T - galaxies: individual: NGC 4527 distance scale

\section{Introduction}

A precondition for the successful use of Ia supernovae at high redshifts for constraining cosmological models is the understanding of low-redshift supernovae, in particular their luminosities and their dependence on lightcurve parameters such as the decline-rate (Phillips 1993). Besides the decline rate, it is also the colour at maximum, for which one has to correct (Tripp 1998; Drenkhahn \& Richter 1999). The Calán-Tololo sample (Hamuy et al. 1996) and the Harvard sample (Riess et al. 1999) demonstrated that most Ia supernovae, after appropriate corrections, exhibit a surprisingly high degree of uniformity in their maximum brightness.

A few supernovae, however, seem to deviate in the sense that they are either too bright or too dim. Since

Send offprint requests to: T. Richtler,

e-mail: tom@coma.cfm.udec.cl

* Based on observations with the NASA/ESA Hubble Space Telescope, obtained at the Space Telescope Science Institute, which is operated by AURA, Inc., under NASA contract NAS $5-26555$.

$\star \star$ Gemini Science Fellow. at high redshifts, selection effects towards the bright end of the supernovae luminosity distribution are probably present, the apparently overluminous supernovae are of particular interest. Obviously, the distance to the host galaxy is the key parameter. For example, in the case of SN 1994D in NGC 4526, it could be shown that this apparently overluminous Ia event took place at a shorter distance than initially assumed, and thus its luminosity is consistent with other type-Ia SNe (Drenkhahn \& Richtler 1999).

Another more extreme example of overluminosity is SN 1991T in NGC 4527. Besides its allegedly high luminosity, it also exhibited spectroscopic peculiarities. (Fischer et al. 1999) discuss the present knowledge about SN 1991T and also offer an interpretation of these abnormal spectral features. Their analysis suggested that SN 1991T was a super-Chandrasekhar explosion following the merging of two white dwarfs.

Although there is general agreement that NGC 4527 is located on the near side of the Virgo cluster, there is no individual distance determination besides a Tully-Fisher distance, where it is known that large deviations can occur. Fischer et al. (1999) assumed its distance to be equal 
to the distance of two neighboring spiral galaxies, which also have been hosts for Ia SNe, NGC 4496 (SN 1960F), and NGC 4536 (SN 1981B).

The aim of our paper is to present an individual distance to SN 1991T, based on surface brightness fluctuations in the bulge of NGC 4527, which is more accurate than what is available in the literature.

Before we present our method of distance determination and its result, we recall briefly what is known about the distance of this galaxy group and the luminosities of the respective Ia SNe.

\subsection{What is known about the distances of NGC 4527 and NGC 4536?}

NGC 4536, the host of SN 1981B, has a more or less well established distance, based on the PL-relation for Cepheids observed by HST (Saha et al. 1996a; Saha et al. 1996b). However, the Cepheid data are to a minor degree subject to interpretational manipulation, depending on details of the data reduction and also of the selection of Cepheids used for the distance determination. Saha et al. (1996b), for instance, quote a distance modulus to NGC 4536 of $31.07 \mathrm{mag}$, while Gibson et al. (2000) give $30.95 \pm 0.23$ mag.

Tully-Fisher (TF) distances have been measured both for NGC 4527 and for NGC 4536. If we use the new TF calibrations given by Sakai et al. (2000) and the TF data given by Pierce (1994), we then get distance moduli (averaged over $B, R, I$ ) of 30.78 and 30.80 for NGC 4527 and NGC 4536, respectively. Pierce (1994) himself quotes 30.6 and 30.5, respectively. The differences are consistent with the dispersion of the TF relation which is about 0.3-0.4 mag. In this sense, the TF data are in agreement with the Cepheid distance of NGC 4536. Moreover, the TF distances cannot support any distance difference between NGC 4527 and NGC 4536 and it appears reasonable to assume a distance modulus of $30.95 \pm 0.23 \mathrm{mag}$ for NGC 4527 as well.

\section{Revised relations between SN la maximum brightness, decline rate and colour}

It is now clear that the intrinsic maximum brightness of SNe Ia vary by approximately 1 mag. Using both the "Calán-Tololo" sample (Hamuy et al. 1996) and the Harvard sample (Riess et al. 1999), we below present handy relations between maximum brightness (extinction corrected), decline rate and colour for the $B, V$, and $I$ bands. We define the colour as the difference between the $B_{\max }$ and $V_{\max }$ maximum magnitudes, which is not necessarily the $B-V$ colour at, say, the $B$-maximum. These relations are revised versions of those given in Drenkhahn \& Richtler (1999), which only included the Calán-Tololo sample. A similar analysis, based on the Calán-Tololo sample alone, has been done by Tripp \& Branch (1999). A colour dependence is definitively present, but it is difficult in the individual case to distinguish between an intrinsic red colour and reddening. We also point out that only the galactic foreground reddening can be accounted for, because internal reddenings are not available. However, if reddening dominated the colour dependence of SN luminosities, we would expect distinctly larger colour coefficients in $B$ and $V$.

The coefficients in Table 1 result from a $\chi^{2}$-fit to the data of 41 (24 in the $I$ band) SN Ia from Hamuy et al. (1996) and Riess et al. (1999). The listed values are the best fits to the relation

$$
\begin{aligned}
& m_{\max }+b \cdot\left(\Delta m_{15}-\overline{\Delta m_{15}}\right) \\
& \quad+R \cdot\left(B_{\max }-V_{\max }-\left(\overline{B_{\max }-V_{\max }}\right)\right) \\
& \quad=5 \cdot \log c z+Z
\end{aligned}
$$

with $m_{\text {max }}$ being the foreground extinction corrected magnitudes in $B, V$ and $I, b$ the decline-rate coefficient, $R$ the colour coefficient, and $Z$ the zero-point of the Hubble diagram defined by the merging of both SNe samples. For the determination of the foreground extinction we used the dust maps of Schlegel et al. (1998).

Table 2 lists the photometric parameters for our SNe taken from Hamuy et al. (1996). The reddening values and their uncertainties are those quoted by Fischer et al. (1999).

Using the correction terms for colour and decline-rate, and the Cepheid distance modulus for NGC 4536, one arrives at the corrected absolute maximum magnitudes given in Table 3.

The Calán-Tololo and the Harvard sample demonstrated that there is no observable offset between the corrected absolute magnitudes of $\mathrm{SNe}$ Ia in early-type and late-type galaxies. Within the uncertainty limits, SN 1981B matches the SNe Ia in the Fornax cluster and also to some of the Ia's in other spiral galaxies, although uncertain photometry and uncertain extinction make the comparison more difficult (Drenkhahn \& Richtler 1999). However, the brightness of SN $1991 \mathrm{~T}$ is inconsistent with that of SN 1981B or other supernovae, if the Cepheid distance of NGC 4536 is also adopted for NGC 4527. Table 3 demonstrates that this discrepancy is not very dependent on the adopted reddening, although the error bars overlap, almost exclusively as a result of the reddening uncertainty (see Fischer et al. 1999 for a compilation of the relevant literature).

\section{The distance to NGC 4527 from surface brightness fluctuations}

We measured a new and accurate distance to NGC 4527 using the near-IR surface brightness fluctuation technique. Two 128-second images of NGC 4527 taken with NICMOS on the Hubble Space Telescope were recovered from the archive. These images were taken using the NIC2 camera using the F160W filter, and have a spatial resolution of 0'13 FWHM. The excellent resolution and very low background (approximately $18.9 \mathrm{mag} / \square^{\prime \prime}$ at $\mathrm{F} 160 \mathrm{~W}$ ) make 
Table 1. The table lists the coefficients according to our correction formula (1) for the relation between brightness, decline rate, and colour

\begin{tabular}{cccccc}
\hline Band & $Z$ & $b$ & $R$ & $\overline{\Delta m_{15}}$ & $\overline{B_{\max }-V_{\max }}$ \\
\hline$B$ & $-3.345 \pm 0.051$ & $-0.61 \pm 0.21$ & $-2.53 \pm 0.62$ & 1.237 & 0.0 \\
$V$ & $-3.356 \pm 0.041$ & $-0.66 \pm 0.18$ & $-1.62 \pm 0.53$ & 1.237 & 0.0 \\
$I$ & $-3.118 \pm 0.050$ & $-0.35 \pm 0.19$ & $-1.12 \pm 0.58$ & 1.230 & -0.017 \\
\hline
\end{tabular}

Table 2. This table lists the photometric parameters for SN $1991 \mathrm{~T}$ and SN 1981B SNe according to Hamuy et al. (1996). The $B, V, I$ magnitudes are not extinction corrected. The reddening values and their uncertainties are those quoted by Fischer et al. (1999) and the uncertainty of the reddening of SN $1991 \mathrm{~T}$ reflects the variety of literature values

\begin{tabular}{lllllll}
\hline Host Galaxy & SN & $B_{\max }$ & $V_{\max }$ & $I_{\max }$ & $E(B-V)$ & $\Delta m_{15}$ \\
\hline NGC 4527 & $1991 \mathrm{~T}$ & $11.69 \pm 0.03$ & $11.51 \pm 0.03$ & $11.62 \pm 0.03$ & $0.20 \pm 0.10$ & $0.94 \pm 0.05$ \\
NGC 4536 & $1981 \mathrm{~B}$ & $12.03 \pm 0.03$ & $11.93 \pm 0.03$ & - & $0.10 \pm 0.05$ & $1.10 \pm 0.05$ \\
\hline
\end{tabular}

Table 3. This table lists the corrected absolute maximum magnitudes of the SNe according to (1) and a distance modulus of 30.95, corresponding to the Cepheid distance of NGC 4536. The photometric parameters are taken from Table 2 and are extinction corrected with $A_{B} / E(B-V)=4.1, A_{V} / E(B-V)=3.1$ and $A_{I} / E(B-V)=1.5$ (Rieke \& Lebofsky 1985). To illustrate the effect of a different reddening, we also give for SN 1991T the values for a reddening of $E(B-V)=0.13$ which has been advocated by Phillips et al. (1992)

\begin{tabular}{llcccc}
\hline Host Galaxy & SN & $\mu$ & $M_{B, \max }$ & $M_{V, \max }$ & $M_{I, \max }$ \\
\hline NGC 4527 & $1991 \mathrm{~T}(E(B-V)=0.20)$ & $30.95 \pm 0.09$ & $-19.87 \pm 0.51$ & $-19.85 \pm 0.38$ & $-19.64 \pm 0.17$ \\
NGC 4527 & 1991T $(E(B-V)=0.13)$ & $30.95 \pm 0.09$ & $-19.75 \pm 0.28$ & $-19.74 \pm 0.21$ & $-19.53 \pm 0.11$ \\
NGC 4536 & 1981B & $30.95 \pm 0.23$ & $-19.26 \pm 0.26$ & $-19.25 \pm 0.20$ & - \\
\hline
\end{tabular}

detection of surface brightness fluctuations straightforward in the bulge of NGC 4527.

The calibration and techniques for determining IR SBF distances using NIC2 in the F160W bandpass were developed by Jensen et al. (2000). This calibration relies on direct measurements of SBFs, both in the $I$ and F160W bands, to nearby spirals with well-determined Cepheid distances (Tonry et al. 2000; Jensen et al. 2000). The absolute fluctuation magnitude calibration $\bar{M}=$ $-4.86 \pm 0.05$ (statistical uncertainty only) presented by Jensen et al. (2000) is essentially constant with galaxy colour redward of $(V-I)=1.16$. NGC 4527 has a colour of $(V-I)=1.23 \pm 0.03(\mathrm{~J}$. Tonry and the optical SBF team, private communication) in the region of the galaxy where the SBF analysis was performed. We adopted the Galactic extinction for NGC 4527 from Schlegel et al. (1998) $A_{B}=$ $0.095 \mathrm{mag}$, implying a correction of only $0.01 \mathrm{mag}$ at F160W. Jensen et al.'s calibration also showed excellent agreement between giant ellipticals and the bulges of spiral galaxies, thereby confirming that the calibration applies equally well to the old, smoothly-distributed stellar populations in the centers of spirals as to the giant elliptical galaxies traditionally targeted for SBF studies.

The methods for measuring SBFs are described in several papers (see Blakeslee et al. 1999 for a review); the infrared techniques used here are are described by Jensen et al. (1998) and Jensen et al. (2000). Basically, the discrete nature of stars in galaxies leads to variations in the number of stars imaged by each pixel in the detector because of Poisson fluctuations. The fluctuations in surface brightness are distance-dependent: distant galaxies look smooth compared to nearby ones because the number of luminous stars imaged by each pixel is larger. The variations are convolved with the point-spread function (PSF) of the telescope, in this case the diffraction pattern of HST as imaged by the NIC2 camera. In practice we observe the variations in luminosity from pixel to pixel rather than variations in the number of stars, so surface brightness fluctuations are dominated by the brightest stars in the galaxy. Because the most luminous stars are red giants in old populations, SBFs are approximately 30 times brighter at F160W than in the optical $I$ band.

The initial image processing proceeded with the galaxy images being flattened and combined after a small bias correction was applied to remove mismatches between detector quadrants. During each of the two exposures, the NIC2 array was read multiple times to allow for the temporal rejection of cosmic rays. Additional cosmic rays were corrected when combining the two images. The results are shown in the left panel of Fig. 1. The inner few arcsec of NGC 4527 are choked with dust lanes in the disk, and these were masked prior to proceeding with the SBF analysis. Point sources were also identified and masked. A smooth model of the galaxy luminosity profile was then constructed and subtracted. An image of NGC 4527 with the galaxy subtracted, showing the stellar SBFs, is shown in the right panel of Fig. 1. 
The spatial power spectrum was constructed and fitted to a linear combination of the scaled PSF power spectrum and a constant, white-noise background. The two components are plotted using dashed lines in Fig. 2, and the solid line is their sum. The "fluctuation power" (in electrons/pixel) is the scale factor by which the power spectrum of the normalized PSF must be multiplied to match the data over the range in wavenumbers where the PSF is dominant. The fit shown in Fig. 2 was constructed for wavenumbers between $k=40$ and 100, corresponding to spatial scales between $0 . .32$ and 0 ". 96 , where the PSF component dominates. Lower wavenumbers are not used because the power spectrum is contaminated by extra power from large-scale residuals of the galaxy subtraction; at wavenumbers higher than 100 the white-noise component dominates. The fit between $k=40$ and 100 is excellent across the entire power spectrum, and yields a fluctuation power of $46.8 \pm 2.6$ electrons/pixel in the $256 \mathrm{~s}$ exposure. The fluctuation power is 15.6 times larger than the white-noise component (effectively the $\mathrm{S} / \mathrm{N}$ ratio of the observation).

Once the fluctuation power was determined, the apparent fluctuation magnitude was easily computed and the distance modulus determined using the Jensen et al. (2000) calibration. The apparent fluctuation magnitude of NGC 4527 is $\bar{m}=25.40 \pm 0.07$, where the uncertainty is the statistical uncertainty arising from the power spectrum fit $(0.054 \mathrm{mag})$, the uncertainty in the PSF normalization (0.061 mag), and the uncertainty in the sky background subtraction (0.028 mag), all added in quadrature. As the PSF and sky were not measured explicitly for the observations of NGC 4527, we used the best values and uncertainties from the library of values collected by Jensen et al. (2000). Subtracting the absolute fluctuation magnitude $\bar{M}$ yields a distance modulus of $(m-M)=30.26 \pm 0.09$, corresponding to a distance (good to $5 \%$ ) of $11.3 \pm 0.50 \mathrm{Mpc}$. NGC 4527 is significantly closer than the bulk of the Virgo cluster.

The IR SBF calibration presented by Jensen et al. (2000) is derived from Cepheid distances to a handful of nearby spirals (Ferrarese et al. 2000), and is hence subject to any systematic uncertainties that affect the Cepheid distance scale generally (such as the distance to the LMC or any period-luminosity dependence on metallicity, for example). The distance modulus uncertainty for NGC 4527 quoted here does not include the additional systematic uncertainty from the Cepheid distance (estimated to be $0.16 \mathrm{mag}$ ) scale beyond that incurred by linking IR SBFs to the Cepheid distances.

\section{The comparison with other SNe la}

It is now interesting to compare SN 1991T with other Ia $\mathrm{SNe}$ in spiral and early-type galaxies, for which more or less accurate distances are known from either Cepheids, from globular cluster luminosity functions, or from surface brightness fluctuations. If we keep the coefficients for decline-rate and colour correction fixed and distinguish
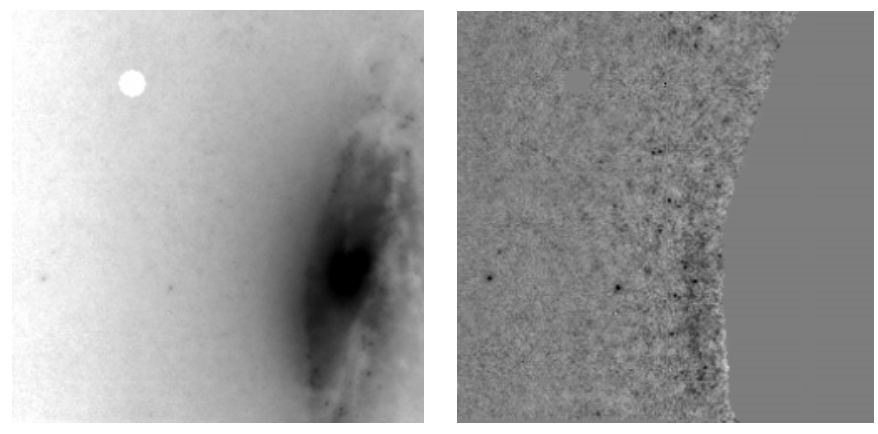

Fig. 1. The NIC2 F160W image of NGC 4527 is shown using a square-root grayscale on the left. The same image with the smooth galaxy profile subtracted is shown on the right, using a linear stretch. Point sources apparent in the galaxy-subtracted image were masked prior to measuring the spatial power spectrum. The field of view is $19^{\prime \prime} .2$ across

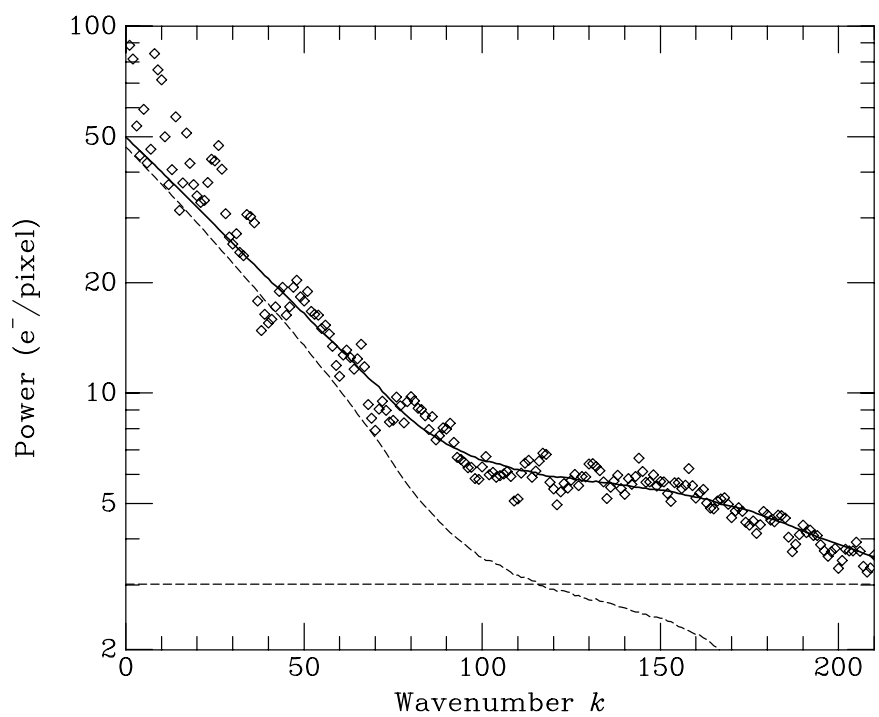

Fig. 2. The azimuthally-averaged spatial power spectrum is shown for the galaxy-subtracted image with point-sources removed. The solid line is the sum of a white-noise (constant) component and the scaled PSF power spectrum (both shown with dashed lines). The scaling of the normalized PSF is the power at $k=0$, and is the surface brightness fluctuations flux from which the fluctuation magnitude is computed

between late-type and early-type host galaxies, the resulting zero-points are for $B, V, I$, respectively (the number of $\mathrm{SNe}$ involved are in parentheses): -3.34 (14), -3.32 (14), -3.04 (9) for early-type host galaxies, and -3.29 (29), $-3.33(29),-3.11$ (16) for late-type host galaxies. It is apparent that there is no significant difference in the corrected absolute magnitudes between SNe Ia in early-type and late-type hosts. We therefore expect the same for $\mathrm{SNe}$ in galaxies whose distances are known.

\subsection{SNe in late-type galaxies}

Gibson et al. (2000) give a compilation of parameters for the $8 \mathrm{SNe}$ Ia in late-type galaxies, which have measured Cepheid distances. We adopt them here since they are also 
the basis for the SFB calibration using Cepheids. To this list, we add SN 1991T. The reddenings used for the extinction calculation are adopted from Phillips et al. (1999). With these data and the correction terms for decline rate and colour presented above, we obtain the corrected absolute magnitudes compiled in Table 4. The uncertainties in the table (in mag) result from a formal error propagation of the different parameters involved. The formally resulting Hubble constant values are also given as the mean of the individual Hubble constant values in the different filters.

Uncertainties in the distances do not necessarily dominate the total uncertainties. Uncertain reddening values and hence uncertain maximum colours make a significant contribution in the case of reddened SNe. This is illustrated in the case of SN 1991T, where the two different reddening values from the literature are adopted (see Table 3). Moreover, in some cases the adopted light curve parameters are more a matter of historical reconstruction than of exact measurements. This is the case for SN 1937C, SN 1960F, and SN 1974G (see van den Bergh 1996 for the relevant literature).

If we skip SN1991T because of the large reddening uncertainty, and SN 1937C, SN 1960F, and SN 1974G from the $\mathrm{SNe}$ with Cepheid distances, we get $-19.15 \pm 0.15$, $-19.15 \pm 0.13,-18.93 \pm 0.17 \mathrm{mag}$ as the weighted means (weight factors are the inverse photometric uncertainties) of the absolute magnitudes for $B, V$, and $I$, respectively. The corresponding Hubble constant values are $68.9 \pm 4.7$, $69.3 \pm 4.1,68.7 \pm 4.1 \mathrm{~km} \mathrm{~s}^{-1} / \mathrm{Mpc}$.

Including all SNe in Table 4, and adopting for SN 1991T the higher reddening value of $E(B-V)=$ $0.2 \mathrm{mag}$, we get $-19.25 \pm 0.13,-19.25 \pm 0.11,-18.93 \pm 0.13$. The corresponding Hubble constant values are $65.8 \pm 3.8$, $65.9 \pm 3.3,68.7 \pm 4.1 \mathrm{~km} \mathrm{~s}^{-1} / \mathrm{Mpc}$.

We thus face the unsatisfactory situation that in spite of the huge effort which has gone into the determination of distances, the resulting Hubble constant is still influenced by the "freedom" of selecting the "right" SNe.

\subsection{SNe la in early-type galaxies}

SNe Ia in S0 or elliptical galaxies are less affected by extinction. There exist some SNe with good photometry in nearby early-type galaxies (Table 5). The Fornax cluster plays a particularly important role by hosting three SNe Ia: SN 1981D and SN 1980N in NGC 1316, and SN 1992A in NGC 1380. Of these, the light curve of SN 1981D has a bad photometry and we skip it here. Then there is SN 1994D in NGC 4526 (Drenkhahn \& Richtler 1999) and SN 1991bg in NGC 4374. The latter has a reputation as a strongly underluminous event.

We adopt the light-curve parameters from the following sources: SN 1980N, SN 1991bg, and SN 1992A from Hamuy et al. (1996), SN 1994D from Drenkhahn \& Richtler (1999). The distance of SN 1994D has been determined by the method of globular cluster luminosity functions and we adopt it also from Drenkhahn \& Richtler (1999) (30.4 \pm 0.3$)$. Della Valle et al. (1998) applied this method to SN 1992A and got $31.3 \pm 0.16$, which is in very good agreement with the distance of Fornax galaxies determined by surface brightness fluctuations (Jensen et al. 1998) and with two of the three late-type galaxies, for which Cepheid distances are available (Ferrarese et al. 2000). There exists no published individual distance yet for NGC 1316, but preliminary results from the analysis of its globular cluster system (Gómez et al., in preparation) indicates very good agreement with SN 1992A and we adopt $30.35 \pm 0.25$. For NGC 4374, we take the distance modulus quoted by Ferrarese et al. (2000) (31.2 \pm 0.1 ), which is in very good agreement with the recent SFB modulus of $(31.17 \pm 0.1)$ by Neilsen \& Tsvetanov (2000).

The weighted average values (weight factors are the inverse uncertainties) of the absolute, corrected magnitudes are $-18.96 \pm 0.18,-18.97 \pm 0.16,-18.66 \pm 0.10$ for $B, V$, $I$, respectively. The corresponding Hubble constant values are $75.3 \pm 6.2,75.4 \pm 5.4,78.1 \pm 3.8 \mathrm{~km} \mathrm{~s}^{-1} / \mathrm{Mpc}$.

Besides SN 1991bg, another strongly underluminous event was SN $1997 \mathrm{cn}$ in the elliptical galaxy NGC 5490 (Turatto et al. 1998). No individual distance determination is known, but the galaxy should be well in the Hubble-flow (heliocentric recession velocity $5008 \mathrm{~km} \mathrm{~s}^{-1}$ ). Therefore, a consistency check is possible by assuming a Hubble constant of $70 \mathrm{~km} \mathrm{~s}^{-1} / \mathrm{Mpc}$ and correspondingly a distance modulus of $34.2 \mathrm{mag}$. Given the light-curve data by Turatto et al. (1998) and assuming zero extinction, we get for the corrected absolute magnitudes in $B, V, I$ $-19.02 \pm 0.57,-19.11 \pm 0.45,-18.85 \pm 0.11$. Thus even such a red SN like SN $1997 \mathrm{cn}$ with $B_{\max }-V_{\max }=0.65$ is consistent with the other SNe. It is striking that in spite of the large errors, especially in $B$, caused by the large and uncertain colour corrections, the absolute magnitudes of SN 1997cn and SN 1991bg agree so well with those of the other SNe. This is perhaps an indication that due to the small number of red SNe, the error of the colour correction is overestimated.

\section{Discussion}

The formal brightness differences between the $\mathrm{SNe}$ in Tables 4 and 5 are $-0.30 \pm 0.25,-0.29 \pm 0.25,-0.31 \pm 0.17$ for $B, V, I$, respectively, if all SNe are taken $(E(B-V)=$ 0.2 in case of SN 1991T). If SN 1991T, SN 1960F, and SN 1974G are skipped, the differences reduce to $-0.21 \pm$ $0.2,-0.19 \pm 0.22$, and $-0.3 \pm 0.2$. Most SNe Ia in late-type galaxies probably have different progenitors from SNe Ia in early-type galaxies, as the distribution of decline-rates suggests: we find only fast decliners in early-type galaxies, but the entire range of decline-rates in late-type galaxies (e.g. Richtler et al. 2000a), which, however, are dominated by slow-decliners.

Also Tripp \& Branch (1999) got a marginally smaller Hubble constant if they select only spirals from the CalánTololo sample in combination with Cepheid calibrated host galaxies. However, the corresponding brightness 
Table 4. This table lists the corrected absolute maximum magnitudes of $8 \mathrm{SNe}$ Ia, whose distances have been measured by Cepheids, together with SN 1991T, for which now the new distance modulus of 30.26 has been used. The distances and light curve parameters are adopted from Gibson et al. (2000), the reddening values, except those for SN 1991T, are from Suntzeff et al. (1999). The uncertainties follow from an error propagation of the various parameters involved. Systematic distance scale uncertainties are not accounted for. The corresponding Hubble constants are the average values (not weighted) of the respective band values

\begin{tabular}{llllll}
\hline Host Galaxy (type) & SN & $M_{B, \max }$ & $M_{V, \max }$ & $M_{I, \max }$ & $H_{0}$ \\
\hline NGC 4527 (Sab) & $1991 \mathrm{~T}(E(B-V)=0.20)$ & $-19.18 \pm 0.52$ & $-19.16 \pm 0.39$ & $-18.95 \pm 0.18$ & $68.6 \pm 11.6$ \\
NGC 4527 (Sab) & $1991 \mathrm{~T}(E(B-V)=0.13)$ & $-19.06 \pm 0.29$ & $-19.05 \pm 0.22$ & $-18.84 \pm 0.18$ & $72.2 \pm 7.3$ \\
NGC 4639 (Sab) & $1990 \mathrm{~N}$ & $-19.09 \pm 0.28$ & $-19.09 \pm 0.27$ & $-18.86 \pm 0.25$ & $71.3 \pm 8.9$ \\
NGC 4536 (Sab) & $1981 \mathrm{~B}$ & $-19.26 \pm 0.27$ & $-19.25 \pm 0.21$ & - & $66.1 \pm 7.4$ \\
NGC 3627 (Sb) & $1989 \mathrm{~B}$ & $-19.19 \pm 0.41$ & $-19.19 \pm 0.37$ & - & $68.0 \pm 12.4$ \\
NGC 3368 (Sab) & $1998 \mathrm{bu}$ & $-19.28 \pm 0.41$ & $-19.27 \pm 0.35$ & $-19.09 \pm 0.28$ & $65.0 \pm 10.5$ \\
NGC 5253 (Im pec) & $1972 \mathrm{E}$ & $-18.90 \pm 0.40$ & $-18.88 \pm 0.37$ & $-18.81 \pm 0.34$ & $76.4 \pm 13.1$ \\
IC 4182 (SAm) & $1937 \mathrm{C}$ & $-19.29 \pm 0.37$ & $-19.27 \pm 0.33$ & - & $65.5 \pm 10.6$ \\
NGC 4496A (SB) & $1960 \mathrm{~F}$ & $-19.55 \pm 0.40$ & $-19.65 \pm 0.37$ & - & $56.4 \pm 10.2$ \\
NGC 4414 (SAc) & 1974G & $-19.58 \pm 0.47$ & $-19.57 \pm 0.39$ & - & $57.1 \pm 11.3$ \\
\hline
\end{tabular}

Table 5. This table lists the corrected absolute maximum magnitudes of SNe Ia in early-type galaxies. Refer the text for data sources

\begin{tabular}{llllll}
\hline Host Galaxy (type) & SN & $M_{B, \max }$ & $M_{V, \max }$ & $M_{I, \max }$ & $H_{0}$ \\
\hline NGC 1380 (S0) & $1992 \mathrm{~A}$ & $-19.01 \pm 0.29$ & $-19.02 \pm 0.27$ & $-18.58 \pm 0.25$ & $76.2 \pm 9.7$ \\
NGC 1316 (S0pec) & $1980 \mathrm{~N}$ & $-19.05 \pm 0.30$ & $-19.05 \pm 0.27$ & $-18.68 \pm 0.25$ & $74.2 \pm 9.5$ \\
NGC 4526 (S0) & $1994 \mathrm{D}$ & $-18.76 \pm 0.35$ & $-18.72 \pm 0.34$ & $-18.46 \pm 0.31$ & $81.5 \pm 12.2$ \\
NGC 4374 (E) & $1991 \mathrm{bg}$ & $-19.03 \pm 0.61$ & $-19.10 \pm 0.49$ & $-18.75 \pm 0.12$ & $73.1 \pm 13.7$ \\
\hline
\end{tabular}

difference is within the statistical uncertainty. Since the combined Calán-Tololo and Harvard samples show that the decline-rate and colour corrections completely account for the intrinsic brightness difference, we are apparently forced to draw the conclusion that any remaining difference after correction is due to a systematic difference in the distances to early- and late-type host galaxies. But even that is not entirely clear. The Fornax cluster distance is dominant for the average Ia brightness in early-type host galaxies. A distance modulus of the Fornax cluster of $31.60 \mathrm{mag}$, as now advocated by Ferrarese et al. (2000), would brighten the two Fornax Ia's in Table 5 by about $0.2 \mathrm{mag}$, which would cause the brightness difference almost to vanish.

On the other hand, the case for $31.35 \mathrm{mag}$ as the Fornax distance modulus is strong (Jensen et al. 1998; Richtler et al. 2000b). Moreover, two of the three Fornax spirals with Cepheid distances from Ferrarese et al. are quoted with distance moduli $31.43 \pm 0.07$ and $31.39 \pm 0.10$ and thus agree very well with the distance based on surface brightness fluctuations and globular cluster luminosity functions. The Hubble constant values from Table 5 also fit well to the value of $77 \mathrm{~km} \mathrm{~s}^{-1} / \mathrm{Mpc}$, emerging from the SFB survey by Tonry et al. (2000). But since some of the supernovae with Cepheid distances still agree within the uncertainties, it would be premature to claim a difference between SFB distances and Cepheid distances.

Based on the sample of spiral host galaxies, Tripp \& Branch (1999) derive $M_{B}=-19.46$ as the colour and decline-rate corrected blue luminosity for SNe Ia. One notes, however, that the mean difference between their adopted distance moduli plus apparent brightness and our values is already $0.3 \mathrm{mag}$. This demonstrates how uncertain the SN Ia brightness in spiral galaxies still is. A deeper discussion, particularly on the Hubble constant and the zero-point uncertainties of the distance scale, is beyond the scope of our paper and we refer the reader to Branch (1998), Gibson et al. (2000), Tonry et al. (2000) and references therein.

Given the above arguments, the present data on supernovae absolute magnitudes does at best marginally support a small difference in the absolute corrected magnitudes of SNe in early-type and late-type galaxies, which, however, is of the order of present uncertainties in the respective absolute distance scales.

We further recall that systematic uncertainties in the distance scale of Cepheids and globular cluster luminosity functions have not yet been included. For example, all distance moduli based on Cepheids use $18.50 \mathrm{mag}$ as the LMC distance modulus, whereas values between 18.2 and $18.7 \mathrm{mag}$ appear in the literature, e.g. Walker (1999).

One can only conclude that progress regarding a further decrease of the uncertainty of the Hubble constant must come from both sources: we need more well observed SNe Ia in nearby spirals and early-type galaxies and a more accurate zero-point determination of the distance scale. 


\section{Summary and conclusions}

By applying the method of surface brightness fluctuations to the bulge of NGC 4527, we measured an accurate distance to this galaxy, which hosted SN 1991T. Our result for the distance modulus is $30.26 \pm 0.09$. With this distance, the absolute maximum magnitudes of SN 1991T, corrected for decline rate and colour, in $B, V, I$ are $-19.18 \pm 0.52,-19.16 \pm 0.39$, and $-18.95 \pm 0.18$, respectively (adopting a reddening of $E(B-V)=0.2 \pm 0.1$. Although the uncertainty is large, mainly caused by the uncertain reddening, there is no reason to conclude that SN $1991 T$ has a peculiarly high luminosity, as the comparison with other SNe shows. Thus the model of a super-Chandrasekhar explosion advanced by Fischer et al. (1999) can not be motivated by the new distance.

Since also underluminous events like SN 1991bg and SN $1997 \mathrm{cn}$ are consistent with the corrected absolute magnitudes of the other SNe, there is now no single SN known, which deviates strikingly, in accordance with the homogeneous samples of the Calán-Tololo and the Harvard sample. Since there is evidence that SNe Ia in late-type and early-type galaxies stem from different parent populations, (see Leibundgut 2000 for a general review on SNe Ia) this may indicate that there is no evolutionary effect as far as the corrected absolute magnitudes are concerned. This is encouraging for the use of SNe Ia in cosmological contexts.

Acknowledgements. We greatly appreciate the help of the NICMOS team, which provided advice, software and the photometric zero point used in the SBF analysis. We also acknowledge the helpful contribution of the optical colour data by the Optical SBF team (J. Tonry, J. Blakeslee, E. Ajhar, and A. Dressler). The NICMOS GTO team was supported by NASA grant NAG 5-3042. J. Jensen acknowledges the support of the Gemini Observatory, which is operated by the Association for Research in Astronomy, Inc., under a cooperative agreement with the National Science Foundation on behalf of the Gemini partnership: the National Science Foundation (USA), the Particle Physics and Astronomy Research Council (UK), the National Research Council (Canada), CONICYT (Chile), the Australian Research Council (Australia), CNPq (Brazil) and CONICET (Argentina). J. Tonry and B. Barris were supported in part by NASA grant GO-07453.0196A.

\section{References}

Blakeslee, J. P., Ajhar, E. A., \& Tonry, J. L. 1999, in PostHipparcos Cosmic Candles, ed. A. Heck, \& F. Caputo,
(Kluwer Academic Publishers, Boston), 181

Branch, D. 1998, A\&AR, 36, 17

Della Valle, M., Kissler-Patig, M., Danziger, J., \& Storm, J. 1998, A\&A, 299, 267

Drenkhahn, G., \& Richtler, T. 1999, A\&A, 349, 877

Ferrarese, L., Mould, J. R., Kennicutt, R. C., et al. 2000, ApJ, 529,745

Fischer, A., Branch, D., Hatano, K., \& Baron, E. 1999, MNRAS, 304, 67

Gibson, B. K., Stetson, P. B., Freedman, W. L., et al. 2000, ApJ, 529, 723

Hamuy, M., Phillips, M. M., Suntzeff, N. B., et al. 1996, AJ, 112,2398

Jensen, J. B., Tonry, J. L., \& Luppino, G. A. 1998, ApJ, 505, 111

Jensen, J. B., Tonry, J. L., Thompson, R., et al. 2000, in preparation

Leibundgut, B. 2000, A\&AR, in press [astro-ph/0003326]

Neilsen, E., \& Tsvetanov, Z. 2000, ApJ, 536, 255

Phillips, M. M. 1993, ApJL, 413, L105

Phillips, M. M., Wells, L. A., Suntzeff, N. B., et al. 1992, AJ, 103,1632

Phillips, M. N., Lira, P., Suntzeff, N. B., et al. 1999, AJ, 118, 1766

Pierce, M. J. 1994, ApJ, 430, 53

Richtler, T., Drenkhahn, G., Gómez, M., \& Infante, L. 2000a, A\&A, submitted

Richtler, T., Drenkhahn, G., Gómez, M., \& Seggewiss, W. 2000b, in From Extrasolar Planets to Cosmology: The VLT opening Symposium, ed. J. Bergeron, \& A. Renzini, ESO Astrophysics Symposia (Springer), 259,

Rieke, G. H., \& Lebofsky, M. J. 1985, ApJ, 288, 618

Riess, A. G., Kirshner, R. P., Schmidt, P., et al. 1999, AJ, 117, 707

Saha, A., Sandage, A., Labhardt, L., et al. 1996a, ApJS, 107, 693

Saha, A., Sandage, A., Labhardt, L., et al. 1996b, ApJ, 466, 55

Sakai, S., Mould, J., Hughes, S. M. G., et al. 2000, ApJ, 529, 698

Schlegel, D. J., Finkbeiner, D. P., \& Davis, M. 1998, ApJ, 500, 525

Suntzeff, N. B., Phillips, M. M., Covarrubias, R., et al. 1999, AJ, 117, 1175

Tonry, J. L., Blakeslee, J. P., Ajhar, E. A., \& Dressler, A. 2000, ApJ, 630, 625

Tripp, R. 1998, A\&A, 331, 815

Tripp, R., \& Branch, D. 1999, ApJ, 525, 209

Turatto, M., Piemonte, A. S. B., et al. 1998, AJ, 116, 2431

van den Bergh, S. 1996, ApJ, 472, 431

Walker, A. 1999, in Post Hipparcos Candles, ed. F. Caputo, \& A. Heck (Dordrecht: Kluwer), 125, 